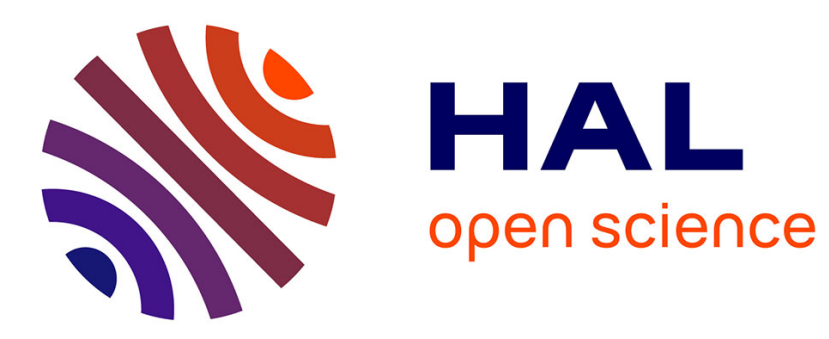

\title{
Understanding Users' Perception of Simultaneous Tactile Textures
}

Yosra Rekik, Eric Vezzoli, Laurent Grisoni

\section{To cite this version:}

Yosra Rekik, Eric Vezzoli, Laurent Grisoni. Understanding Users' Perception of Simultaneous Tactile Textures. Mobilehci, Sep 2017, Vienne, Austria. 10.1145/3098279.3098528 . hal-01578729

\section{HAL Id: hal-01578729 \\ https://inria.hal.science/hal-01578729}

Submitted on 29 Aug 2017

HAL is a multi-disciplinary open access archive for the deposit and dissemination of scientific research documents, whether they are published or not. The documents may come from teaching and research institutions in France or abroad, or from public or private research centers.
L'archive ouverte pluridisciplinaire HAL, est destinée au dépôt et à la diffusion de documents scientifiques de niveau recherche, publiés ou non, émanant des établissements d'enseignement et de recherche français ou étrangers, des laboratoires publics ou privés. 


\section{Understanding Users' Perception of Simultaneous Tactile Textures}

\author{
Yosra Rekik \\ University of Lille Sci. \& \\ Tech, CNRS, INRIA \\ yosra.rekik@inria.fr
}

\author{
Eric Vezzoli \\ University of Lille Sci. \& \\ Tech, CNRS, INRIA \\ eric@gotouchvr.com
}

\author{
Laurent Grisoni \\ University of Lille Sci. \& \\ Tech, CNRS, INRIA \\ laurent.grisoni@univ-lille1.fr
}

\begin{abstract}
We study users' perception of simultaneous tactile textures in ultrasonic devices. We investigate how relevant is providing the user with different complementary and simultaneous textures with respect to the different fingers that can be used to touch the surface. We show through a controlled experiment that users are able to distinguish the number of different textures independently of using fingers from one or two hands. However, our findings indicate that users are not able to differentiate between two different textures, that is to correctly identify each of them, when using fingers from the same hand. Based on our findings, we are then able to outline three relevant guidelines to assist multi-finger tactile feedback ergonomic and devices design.
\end{abstract}

\section{Author Keywords}

Tactile feedback; perception simultaneous textures; ultrasonic device; experiment; finger; one-handed; two-handed.

\section{ACM Classification Keywords}

H.5.2. Information Interfaces and Presentation (e.g. HCI): User Interfaces

\section{INTRODUCTION}

Touch interaction is one of the most dominant ways users interact due to the broad range of interaction styles offered by multi-touch devices, but also to the pervasiveness of touchenabled devices. Touch interaction can be enhanced with a tactile feedback that provides stimulation when touching the surface with the subsequent aim of integration in the mobile world. For example, electrovibration technologies [2, 14] enhances the friction between the finger and the interaction surface while ultrasonic technologies reduce the friction through the "squeeze film effect" $[1,3,10,15])$. In the remainder of this paper, we are specifically interested in the latter type.

The current practice of tactile feedback surface design has outlined several guidelines to assist practitioners in how users feel and identify objects through the touch sense $[11,8,13]$.

Permission to make digital or hard copies of all or part of this work for personal or classroom use is granted without fee provided that copies are not made or distributed for profit or commercial advantage and that copies bear this notice and the full citation on the first page. Copyrights for components of this work owned by others than ACM must be honored. Abstracting with credit is permitted. To copy otherwise, or republish, to post on servers or to redistribute to lists, requires prior specific permission and/or a fee. Request permissions from Permissions@acm.org.

MobileHCI '17, September 04-07, 2017, Vienna, Austria

Copyright () 2017 ACM ISBN 978-1-4503-5075-4/17/09 ...\$15.00.

http://dx.doi.org/10.1145/3098279.3098528
Different applications have then been emerged to improve the immersive experience during interaction [9]; or to offer accessible interaction design for people with impairment [6]; or to strengthen the interaction [10]. However, and despite this positive evolution, tactile feedback interaction with most current devices is still limited to a one unique tactile feedback for the whole device. In fact, touching the surface with one finger or multiple fingers is still implemented to provide the same feedback for the users even when fingers are touching different textures (e.g., the index finger is touching the sea while the middle finger is touching the sand), or when different commands are attributed to different fingers or chords in contact with the surface [5]. This can be explained by today's lack of hardware capability of providing different simultaneous tactile textures. We also believe that the community lacks an in-depth understanding of users' perception of simultaneous tactile textures. Among other fundamental questions, one can for instance ask the following: are users able to perceive simultaneous but different textures on different fingers? Are users able to identify different textures when using fingers from the same hand? Does the number of hands matters in such a setting?

In this context, we argue that users' perception of simultaneous textures is an important factor for tactile feedback surface design that has been little explored so far and, consequently, is little understood. Two limited studies addressing similar challenges have been conducted for braille [16] and vibrotactile [4] devices. Ziat et al [16] evaluated whether participants are able to identify dots displacements and Catala et al. [4] asked participants to determine the location of a single object within the exploration area. In these both studies, the ability of users to identify simultaneous but different textures; each one sensed by a different finger in contact with the surface, has not been evaluated. Additionally, the findings there-in, although of valuable contribution, can not be mindlessly applied to ultrasonic devices which, in contrast to braille and vibrotactile devices, provide considerably different sensations [15]. Besides, vibrotactile devices suffer from a decrease in perception accuracy when sliding repeatedly [7]. In this paper, we examine users' perception of a pair of textures in ultrasonic devices, and we provide the community with the first investigations on the subject and the potential implications in terms of tactile feedback ergonomic and devices design.

The contributions of this work are: (1) through a controlled experiment, we deliver a comprehensive comparison between the use of different finger(s) conditions to perceive a pair of 


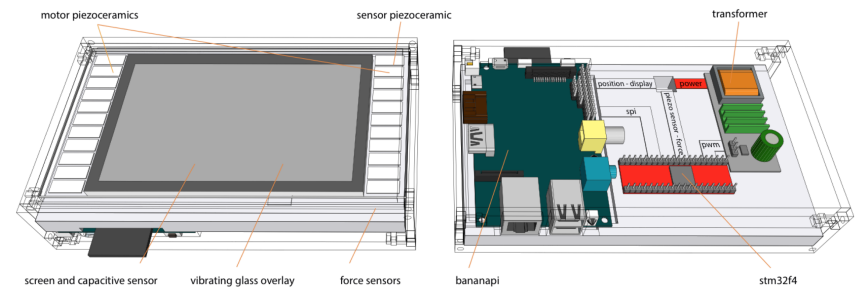

Figure 1: The E-vita device.

textures, (2) we report novel findings on user perception of simultaneous textures, mainly by showing that although users can distinguish the number of different textures, they are not able to correctly identify them when using only one hand, and (3) we derive three guidelines for multi-finger tactile feedback ergonomic and devices design.

\section{EXPERIMENT}

We conducted an experiment to evaluate whether simultaneous textures are consistently perceived by users. We are mainly interested in evaluating whether users are able or not to perceive simultaneous but different textures; each one is sensed by a different finger touching the surface. Moreover, we are interested in evaluating whether the user perception of simulatneous textures can be related to the number of hands touching the surface.

\section{Participants}

15 participants (3 females) volunteered to take part into our experiment. Their age was between 20 and 34 years (mean=26.35, s.d=3.67). All participants were right handed.

\section{Method}

Providing simultaneous but different tactile feedback embedded in the same surface is not available yet. We, therefore, limit the experiment to two textures. The rationale is that if no effect was found with this setting, it would be likely that no such effect exists in more complicated settings. We then, setup two simultaneous tactile feedback surfaces (E-vita [15]); one used as a server while the second is used as a client; that together allow us to deliver two fingertip tactile feedback; each one is provided by a different surface.

The E-VITA device [15] (Figure 1) is based on a banana pi (Shenzhen LeMaker Technology Co. Ltd, China) single board computer featuring a $1 \mathrm{GHz}$ ARM Cortex-A7 dual-core CPU with $1 \mathrm{~GB}$ of ram working in parallel. It is equipped with a 5 inches LCD display including a cheap capacitive sensor which allows a sampling frequency of $50 \mathrm{~Hz}$, similar to the capabilities of commercial mobile devices.

On top of the display, a $154 \times 81 \times 1.6 \mathrm{~mm}$ glass plate, resonating at $60750 \mathrm{~Hz}$ with a half wavelength of $8 \mathrm{~mm}$, is fixed and actuated by twenty $14 \times 6 \times 0.5 \mathrm{~mm}$ piezoelectric cells. A power electronic circuit converts a $12 \mathrm{~V}$ DC voltage source into an AC voltage, controlled in amplitude and frequency and supplied to the piezoelectric cells. A microcontroller (stm32f4, STMicroelectronics, France) runs in parallel with the banana pi and adjusts the circuit's control signals in order to obtain the required vibration amplitude of the plate. For that purpose,

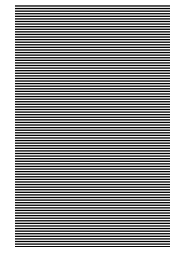

(1) Densest

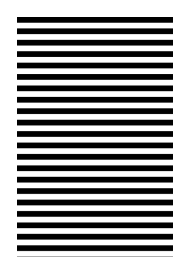

(2) Dense

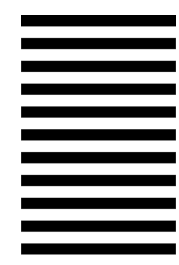

(3) Sparse

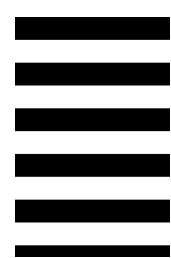

(4) Sparsest
Figure 2: The four visual representation of the experimented textures numbered from left to right (1-4)

two additional piezoelectric cells are used as vibration sensors, and the vibration amplitude is controlled in a closed loop by the microcontroller. The reference value is sent by the banana pi to the microcontroller through a Serial Peripheral Interface (SPI) connection.

In this work, a texture (see Figure 2) refers to a sequence of periodic tactile feedback [13] such that the period to be reproduced inside the texture can be formed by some specific signal (periodic, structured noise, micro-geometry extracted, etc.). We then consider four different tactile textures. We encode the different textures with respect to different texture densities by considering the following spatial periodicity: Densest -1.2 $\mathrm{mm}$; Dense - $5 \mathrm{~mm}$; Sparse - $10 \mathrm{~mm}$ and Sparsest $-20 \mathrm{~mm}$ (see Figure 2). High friction was associated to black color and low friction was associated to white color. The set of considered textures follows the set used by Rekik et al. [13]; for which users are shown to be able to distinguish them independently on the finger velocity; as well as current studies on user perception of tactile textures (e.g., [15, 8, 12]). The set of considered textures is also limited to one-dimensional textures in order to simplify the task.

To render a given texture we use the so-called Localized Haptic Texture (LHT) rendering technique [13]. LHT separates the tactile rendering in two different processes: first, the finger position is retrieved from the hardware, and the corresponding texture is selected through a search in a grid of taxels (tactile element). The taxel texture is then rendered locally by defining only one period of the texture and then repeated it in a loop at a rate that depends on finger's velocity. LHT was shown to provide a high-fidelity between the tactile texture and its visual representation. For instance, LHT leads to the highest level of quality of tactile rendering for both dense and sparse textures. In addition, the performance benefits of LHT were consistent across different finger velocities.

\section{Design}

A within-subjects design was used. The independent variables were the finger or chord used to contact the surfaces and the pair of textures to identify. We employ the same contact conditions than Ziat et al [16] and Catala et al. [4]. Contact covered then a specific finger (the index finger from the dominant hand), one-handed chord (index and middle fingers from the dominant hand) and two-handed chord (the two index fingers). The rational of comparing the one finger condition to the chord conditions is to consider the finger condition as the baseline of what textures could be distinguished when using 


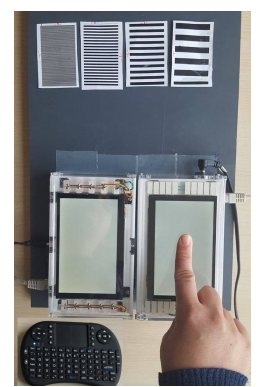

(1) Finger

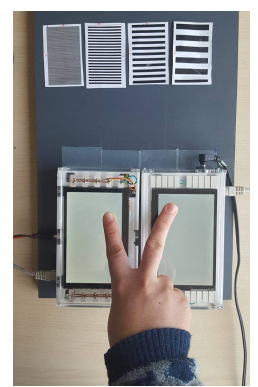

(2) One-handed

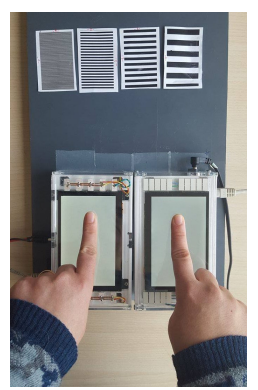

(3) Two-handed
Figure 3: Set-up for the three contact conditions.

one finger sequeltial exploration of the pair of textures. The experiment was, also, limited to these three contact conditions in order to reduce the duration of the experiment. We consider all the ordered pairs of the four textures aforementioned (see Figure 2), where each texture is displayed on one different surface, hence investigating 16 pair of textures (12 pairs of different textures and 4 pairs of similar ones).

\section{Task}

The task required participants to use a specific finger or chord to contact the surfaces. Participants were then asked to move their finger(s) on the two surfaces from top to bottom and inversely to perceive the pair of textures, without a starting finger position or time restrictions. In the finger condition, participants were asked to move their index finger from the dominant hand on the first surface than on the second surface and so on (see Figure 3.1). Participants had the total liberty in choosing the surface they sensed first as well as how to proceed to explore the whole texture. In the one-handed condition, participants were asked to move simultaneously and similarly (the same movement direction and velocity) the index and middle fingers from the dominant hand such that the index finger explores the left surface while the middle finger explores the right surface (see Figure 3.2). In the two-handed condition, participants were asked to move simultaneously and similarly their index fingers such that the index finger from the right hand explores the right surface while the index finger from the left hand explores the left surface (see Figure 3.3). A trial ended once the participant press on the "enter" button on the external keyboard. The participants were then asked the following questions: (1) "Did you perceive the same texture on the two surfaces or two different textures"; (2) "I felt confident in my ability to determine the number of textures"; (3) "Please identify the two perceived textures, left texture then right texture" and (4) "I felt confident in my ability to identify the visual representation of the two textures". Both confidence scales solicited ordinal responses from $1=$ strongly disagree to $10=$ strongly agree.

No visual feedback was shown on the surfaces, only tactile feedback was sent to the participant. However, four sheets of paper illustrating the visual of the four tactile textures were given to participants (see Figure 3). Each visual is given a number between 1 and 4 . In addition, as the Evita device makes noise when alternating high and low frictions, the par-

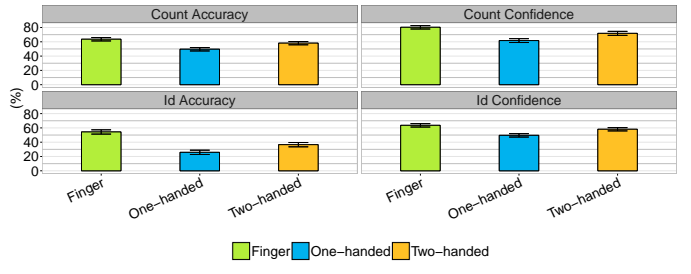

Figure 4: Mean (s.d) of the dependent variables (count accuracy and id accuracy) and the percentage of the confidence responses.

ticipants were equipped with noise reduction headphones to avoid any bias. Tactile feedback was hence made eyes-free since the participants were not able to see any visual (nor to hear any audio) rendering on the surface.

\section{Procedure}

After answering a demographic questionnaire, the experiment task was explained along with the additional requirement of using a specific finger or two-finger chord. The participant then began the experiment. The experimental trials were administered as 3 blocks of 48 trials, each block sharing a contact condition. Inside each block, 48 trials (16 pair of textures $\times 3$ repetitions) were randomly presented to each participant $-\mathrm{a}$ total of 144 trials per participant. Participants could practice for an unlimited time at the beginning of each block.

After each block, participants responded to 5-point Likertscale questions (strongly disagree to strongly agree): i) I identified well, ii) I accomplished the task rapidly; ii) I needed to concentrate to identify well; iv) I felt frustrated; v) I felt confident in my ability to identify the textures and vi) I enjoyed interacting with the device. After terminating all the trials, participants were asked to rank the three contact conditions according to their preferences. The average duration of the experiment was 45 mins.

\section{RESULTS}

The dependent measures are the count accuracy, id accuracy and time. The count accuracy and id accuracy are defined as the proportion of correct identifications of respectively the number of different textures and the visual representations of the pair of textures. The time is defined as the time that a user takes from starting an identification until pressing the "enter" button. The count accuracy and id accuracy obviously provide a sound measures of whether the users are able or not to perceive a pair of textures. The time is more subjective and can only provide an estimation of how difficult might be the identification for participants. We also analyzed subjective responses. All analyses are repeated-measures ANOVA. Tukey tests are used post-hoc when significant effects are found.

Due to a technical issue, the data of two participants were not completely logged. In the following, we report results for the dependent variables and subjective results for 13 participants.

\section{Count Accuracy}

In average, we found that participants are relatively able to identify the number of textures perceived for the three contact 
conditions (see Figure 4). We found significant main effects of contact $\left(F_{2,24}=28.99, p<.0001\right)$ and textures $\left(F_{15,180}=9.18\right.$, $p<.0001$ ) on count accuracy. Without surprise we found that the count accuracy was significantly higher for finger than one-handed by $43.5 \%(p<.001)$ and two-handed by $21.33 \%$ $(p<.01)$. Post-hoc comparison also revealed that count accuracy was significantly lower with one-handed than two-handed $(p<.05)$. Interestingly, there was no significant contact $\times$ textures interaction $(p=.755)$, suggesting that the benefits of finger and two-handed are consistent across different textures.

Immediately after entering the number of textures perceived, participants rated their score of confidence. Friedman tests revealed a significant effect of contact on confidence rating $\left(\chi^{2}(2)=135.44, p<.0001\right)$ with participants significantly more confident when using finger (respectively two-handed) than when using both chord conditions (respectively onehanded $)(p<.0001)$.

\section{Id Accuracy}

The overall performance is modest for the three contact conditions and especially poor for the one-handed condition (see Figure 4). On average, when using fingers from the same hand, our participants were successful at identifying the textures associated to the perceived tactile feedback in only $25.81 \%$ of all trials. We also found significant main effects of contact $\left(F_{2,24}\right.$ $=28.51, p<.0001)$ and textures $\left(F_{15,180}=6.43, p<.0001\right)$ on id accuracy. Post-hoc comparison revealed that finger is significantly more accurate than both Chord conditions $(p<.01)$ by $110.49 \%$ for one-handed and $48.68 \%$ for two-handed. Twohanded was also significantly more accurate than one-handed $(p<.05)$. We also found that the most easily differentiated pairs of textures are the pair of textures composed by the same densest textures (P1) $($ mean $=72.64$, s.d $=10.69 \%)$ followed by the pair of textures composed by the densest and the sparsest textures $(\mathrm{P} 2)(\mathrm{mean}=53.84$, s.d $=11.15 \%)$. Post-hoc comparison confirms that these pairs are the most perceived $(p<.05)$. Interestingly, there was no significant contact $\times$ textures interaction ( $p=.14)$, suggesting that: (1) the benefits of finger and two-handed are consistent across different textures; and (2) the benefits of P1 and P2 are consistent across different contact conditions.

Immediately after determining the number of textures perceived, participants rated their score of confidence. Friedman tests revealed a significant effect of contact on confidence rating $\left(\chi^{2}(2)=130.05, p<.0001\right)$ with participants significantly more confident when using finger (respectively, twohanded) than when using both Chord conditions (respectively one-handed $)(p<.0001)$.

\section{Time}

There were no significant main effects of contact $(\mathrm{p}=.45)$ nor of textures ( $p=.43$ ) on time with no significant contact $\times$ textures interaction $(p=.41)$, suggesting that the time needed to identify a pair of textures is independent on the contact used and on textures type $($ mean $=17423, \mathrm{~s} . \mathrm{d}=2832 \mathrm{~ms})$.

\section{Subjective Results}

We recall that participants were asked to rank the contact conditions after completing the corresponding trial blocks.

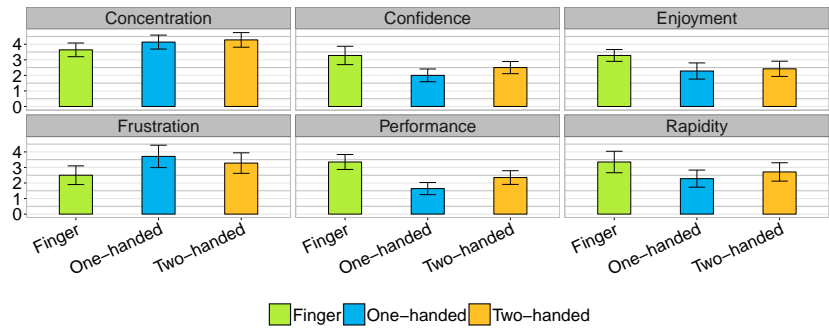

Figure 5: Mean (s.d) questionnaire responses, with 1=strongly disagree, and $5=$ strongly agree.

Overall, finger was ranked first $100 \%$ of the time, two-handed was ranked $77 \%$ second and $23 \%$ third while one-handed was ranked second $23 \%$ and third $77 \%$.

Participants were also asked to rate each contact condition (see Table 5). Friedman tests revealed a significant effect of contact on performance, frustration, confidence and enjoyment. Post-hoc tests using Bonferroni correction showed that one finger was significantly better performing and implies more confidence than both Chord conditions $(p<.01)$ while being less frustrating $(p=.02)$ and more enjoyable $(p=.03)$ than one-handed.

We correlate these findings with participants comments that felt that using one finger is easier and demands less mental effort than when using both chord conditions. Some quotes are: "Using one finger is easier as I can focus on one texture each time" and "As I don't success to identify the textures when using two fingers, ..., I prefer to sense the textures using one finger with sequential exploration". On the other hand, participants felt that it might be more sophisticated to operate in the two-handed condition compared to the finger condition, but, is much more convenient than the one-hand condition. One participant witnessed: "I prefer using two hands to perceive different sensations as it is easier than when I use fingers from the same hand". All participants felt that the one-handed condition is difficult and frustrating. One participant said: "It is really annoying... I am unable to identify the textures" and another said: "I feel very confused, I perceived the feedback in a very fuzzy way", then added "I feel like if the densest texture is dominant... in fact, the densest texture is more perceived and so the second one seems like if it is the same", while other participant said "I am unable to differentiate between the textures... I should be very concentrated".

\section{Methodology for Identifying the Pair of Textures}

To better understand how participants were performing, we report here-after the different strategies elaborated by participants in order to identify the textures and the number of textures composing it; which is the by-product of the discussions that followed each contact block.

\section{Strategies in the Finger condition}

Based on participants comments, we state the following:

- Determining the number of textures. All participants except two, first try to figure out how many textures they are able to perceive. For this purpose, two main strategies are 
used: (1) moving successively the finger on one surface and then on the second one in order to determine if it feels the same or not and (2) exploring many times the first surface and then experience the second surface to determine whether it exposed the same texture or not. If the two surfaces are judged as containing two different textures, then participants explored each surface separately to identify the texture using the next strategies. The two remaining participants proceeded by first identifying the texture on one surface and then the second texture in the second surface to determine whether there are one or two textures.

- Counting the number of high frictions. Most of participants counted the number of high frictions and then tried to match the tactile feedback position with its visual.

- Searching the densest and sparsest textures. Some participants noticed that, in addition, "densest and sparsest textures were helpful to classify the texture" by eliminating the textures that do not contain those types and inversely.

\section{Strategies in the Chord conditions}

Two main strategies are used:

- Concentrating on one finger each time. Most of participants tried to concentrate on one finger at once in order to identify the corresponding texture.

- Searching the densest and sparsest textures. Some participants tried to identify the densest or the sparsest texture in the corresponding pair, which then helped them identifying the second texture.

Although these two strategies are used in both chord conditions, all our participants confirmed that they were relatively unable to identify the whole textures in the one-handed condition as they felt that "the densest texture is dominant", and hence, does not help them identify the pair of textures. Interestingly, some quotes are: "using fingers from the same hand requires to be very concentrated as the same part of the brain is used to control the two fingers. However, with two hands, there are two parts of the brain each one can control a different hand, so it is possible to concentrate on one finger at a time".

\section{DISCUSSION \& DESIGN GUIDELINES}

Our key finding is that the users are relatively able to identify the number of different textures composing a pair of textures when using two fingers either from the same hand or two hands. However, they are unable to identify different textures especially when using one hand. It is also important to note that using one finger with sequential exploration of the pair of textures leaded to the highest level of performance, increased the confidence and decreased the frustration over synchronous multi-finger exploration. Finally, our analysis suggests that the time needed to identify a pair of textures is independent of the finger or chord in contact with the surface and the textures.

Informed by our experimental findings, we outline three relevant guidelines for designing multi-tactile feedback ergonomic and devices design: (a) Where possible, privilege one finger sequential exploration of tactile textures over multi-finger synchronous exploration as it is more accurate and increases the confidence.

(b) The same texture should be provided for fingers from the same hand. Indeed, our participants are not able to perceive correctly more than one texture by hand. Importantly, our participants felt that perceiving more than one texture by hand is very mentally demanding and frustrating.

(c) Simultaneous different textures should be limited to two textures by user; each one is perceived by a different hand. Our participants prefer two-handed over one-handed as it is mentally more manageable.

\section{CONCLUSION}

In this paper, we conducted the first investigation on users' perception of simulateous textures in the context of ultrasonic haptic devices. We showed the potential performance limitations of simultaneously perceiving different tactile textures. We hope that our results will help hardware engineers to design accurate dedicated multi-tactile feedback touch-screens, and will prove useful to researchers and practitioners for improving user's experience through the design of enhanced multi-tactile feedback techniques and interfaces.

\section{ACKNOWLEDGMENTS}

This work was partially funded by European funds for economic development (ERDF), ANR (equipex IRDIVE), and CPER Mauve.

\section{REFERENCES}

1. Amberg, M., Giraud, F., Semail, B., Olivo, P., Casiez, G., and Roussel, N. STIMTAC: A Tactile Input Device with Programmable Friction. In Proc. of UIST, ACM (2011), 7-8.

2. Bau, O., Poupyrev, I., Israr, A., and Harrison, C. Teslatouch: Electrovibration for touch surfaces. In Proc. of UIST, ACM (2010), 283-292.

3. Casiez, G., Roussel, N., Vanbelleghem, R., and Giraud, F. Surfpad: Riding towards targets on a squeeze film effect. In Proc. of CHI, ACM (2011), 2491-2500.

4. Catala, A., Miguel, O., Molina, J. P., and Gonzalez, P. Involving multiple fingers in exploring a haptic surface: an evaluation study. The Visual Computer (2016), 921-932.

5. Gupta, A., and Balakrishnan, R. Dualkey: Miniature screen text entry via finger identification. In Proc. of CHI, ACM (2016), 59-70.

6. Israr, A., Bau, O., Kim, S.-C., and Poupyrev, I. Tactile feedback on flat surfaces for the visually impaired. In Proc. of CHI EA, ACM (2012), 1571-1576.

7. Kaczmarek, K. A., Nammi, K., Agarwal, A. K., Tyler, M. E., Haase, S. J., and Beebe, D. J. Polarity effect in electrovibration for tactile display. IEEE Transactions on Biomedical Engineering 53, 10 (Oct 2006), 2047-2054. 
8. Kalantari, F., Grisoni, L., Giraud, F., and Rekik, Y. Finding the minimum perceivable size of a tactile element on an ultrasonic based haptic tablet. In Proceedings of the 2016 ACM on Interactive Surfaces and Spaces, ISS '16, ACM (2016), 379-384.

9. Kuchenbecker, K. J., Fiene, J., and Niemeyer, G. Improving contact realism through event-based haptic feedback. Proc. of VCG, IEEE (2006), 219-230.

10. Levesque, V., Oram, L., MacLean, K., Cockburn, A., Marchuk, N. D., Johnson, D., Colgate, J. E., and Peshkin, M. A. Enhancing physicality in touch interaction with programmable friction. In Proc. of CHI, ACM (2011), 2481-2490.

11. MacLean, K. E. Haptic interaction design for everyday interfaces. Reviews of Human Factors and Ergonomics (2008), 149-194.

12. Potier, L., Pietrzak, T., Casiez, G., and Roussel, N. Designing tactile patterns with programmable friction. In
Actes De La 28IÈMe ConfÉRence Francophone Sur L'Interaction Homme-Machine, IHM '16, ACM (New York, NY, USA, 2016), 1-7.

13. Rekik, Y., Vezzoli, E., Grisoni, L., and Giraud, F. Localized haptic texture: A rendering technique based on taxels for high density tactile feedback. In Proc. of CHI'17. ACM,

14. Senseg. Inc. http://senseg.com/, 2016.

15. Vezzoli, E., Sednaoui, T., Amberg, M., Giraud, F., and Lemaire-Semail, B. Texture rendering strategies with a high fidelity capacitive visual-haptic friction control device. In Proc. of EuroHaptics, Springer (2016), 251-260.

16. Ziat, M., Hayward, V., Chapman, C. E., Ernst, M. O., and Lenay, C. Tactile suppression of displacement. Experimental brain research (2010), 299-310. 